\title{
ON SOME INEQUALITIES OF S. BERNSTEIN AND W. MARKOFF FOR DERIVATIVES OF POLYNOMIALS*
}

\author{
A. C. SCHAEFFER AND R. J. DUFFIN
}

A well known inequality on the derivatives of polynomials is that of S. Bernstein. $\dagger$

BeRnSteIn's THEOREM. Suppose that $f(x)$ is a polynomial of degree $n$ or less, and that in the interval $(-1,1)$

Then

$$
|f(x)| \leqq 1 \text {. }
$$

$$
\left|f^{\prime}(x)\right|^{2} \leqq \frac{n^{2}}{1-x^{2}}, \quad-1 \leqq x \leqq 1,
$$

and the equality can occur only if $f(x) \equiv \gamma T_{n}(x),|\gamma|=1, \ddagger$ where $T_{n}(x)$ is the nth Tchebychef polynomial.

The extension of this theorem of Bernstein to the higher derivatives plays an important role in this paper. Thus, if $f(x)$ satisfies the conditions given in Bernstein's theorem, we obtain the inequality

$$
\left|f^{(p)}(x)\right|^{2} \leqq\left(\frac{d^{p}}{d x^{p}} \cos n \theta\right)^{2}+\left(\frac{d^{p}}{d x^{p}} \sin n \theta\right)^{2}, \quad x=\cos \theta,
$$

for $x$ in $(-1,1)$. Using this inequality we are able to give a simple proof of W. Markoff's theorem, $\S$ which states that under the conditions of Bernstein's theorem

$$
\left|f^{(p)}(x)\right| \leqq \frac{n^{2}\left(n^{2}-1^{2}\right)\left(n^{2}-2^{2}\right) \cdots\left(n^{2}-(p-1)^{2}\right)}{1 \cdot 3 \cdot 5 \cdot \cdots \cdot(2 p-1)},-1 \leqq x \leqq 1 .
$$

* Presented to the Society, April 8, 1938.

$\dagger \mathrm{S}$. Bernstein, Sur l'ordre de la meilleure approximation des fonctions continues par des polynômes de degré donné, Mémoires de l'Académie Royale de Belgique, (2), vol. 4 (1912), pp. 1-104. M. Riesz, Eine trigonometrische Interpolationsformel und einige Ungleichungen fiur Polynome, Jahresbericht der Deutschen Mathematiker-Vereinigung, vol. 23 (1914), pp. 354-368.

$\ddagger \gamma$ stands hereafter for a constant, real or complex, of absolute magnitude 1 .

$\S \mathrm{W}$. Markoff, Über Polynome, die in einem gegebenen Intervalle möglichst wenig von null abweichen, Mathematische Annalen, vol. 77 (1916), pp. 213-258, translated by J. Grossman. The original appeared in Russian in 1892.

G. Szegö, Über einen Satz von A. Markoff, Mathematische Zeitschrift, vol. 23 (1925) pp. 45-61. 
Before proceeding with the proof of these inequalities we find it necessary to establish several lemmas concerning the properties of the Tchebychef polynomial $T_{n}(x)$, and a related function $S_{n}(x)$, (not a polynomial) which are defined by the relations

$$
\begin{aligned}
& T_{n}(x)=\cos n \theta, \\
& S_{n}(x)=\sin n \theta, \\
& x=\cos \theta,
\end{aligned}
$$

$n$ being any positive integer. These functions are independent solutions of the differential equation

$$
\left(1-x^{2}\right) R^{\prime \prime}(x)-x R^{\prime}(x)+n^{2} R(x)=0,
$$

the general real solution of which may be written

$$
R(x)=a T_{n}(x)+b S_{n}(x)=c \cos (n \theta-\alpha), \quad x=\cos \theta,
$$

where $a, b, c$, and $\alpha$ are real constants. Differentiating (2) $p$ times we obtain

(3) $\left(1-x^{2}\right) R^{(p+2)}(x)-(2 p+1) x R^{(p+1)}(x)+\left(n^{2}-p^{2}\right) R^{(p)}(x)=0$,

and this may be written in the equivalent form

(4) $\frac{d}{d x}\left\{\left[1-x^{2}\right]\left[R^{(p+1)}(x)\right]^{2}+\left[n^{2}-p^{2}\right]\left[R^{(p)}(x)\right]^{2}\right\}=4 p x\left[R^{(p+1)}(x)\right]^{2}$.

For $p \leqq n$ the functions $T_{n}^{(p)}(x)$ and $S_{n}^{(p)}(x)$ are particular solutions of (3) and (4).

Let $M_{p}(x)$ be defined by the relation

$$
\begin{aligned}
M_{p}(x) & =\left(T_{n}^{(p)}(x)\right)^{2}+\left(S_{n}^{(p)}(x)\right)^{2} \\
& \equiv\left(\frac{d^{p}}{d x^{p}} \cos n \theta\right)^{2}+\left(\frac{d^{p}}{d x^{p}} \sin n \theta\right)^{2} .
\end{aligned}
$$

Then it is clear that $M_{p}(x)$ also satisfies (4), that is

$$
\frac{d}{d x}\left\{\left(1-x^{2}\right) M_{p+1}(x)+\left(n^{2}-p^{2}\right) M_{p}(x)\right\}=4 p x M_{p+1}(x) .
$$

LEMMA 1. In the open interval $(-1,1), T_{n}^{(p)}(x)$ has $n-p$ zeros all of which are simple, $(p=0,1,2, \cdots, n) ; S_{n}{ }^{(p)}(x)$ has $n+1-p$ zeros all of which are simple, $(p=1,2,3, \cdots, n+1)$; and the zeros of $T_{n}{ }^{(p)}(x)$ and $S_{n}{ }^{(p)}(x)$ separate one another, $(p=1,2,3, \cdots, n)$.

Proof. From the definition (1) we see that $T_{n}(x)$ has $n$ simple zeros in the interior of $(-1,1)$, and since it is a polynomial of degree $n$, these are its only zeros. From Rolle's theorem it follows that 
$T_{n}{ }^{(p)}(x),(p=1,2, \cdots, n)$, has exactly $n-p$ zeros in $(-1,1)$, all of which are simple. From (1) we see that $S_{n}(x)$ may be expressed in the form

$$
S_{n}(x)=T_{n}^{\prime}(x)\left(1-x^{2}\right)^{1 / 2} / n,
$$

from which it follows that $S_{n}(x)$ has $n+1$ zeros in the closed interval $(-1,1)$, and that $S_{n}^{\prime}(x)$ becomes infinite as $x \rightarrow \pm 1$. By successive use of Rolle's theorem it follows that $S_{n}{ }^{(p)}(x)$ must have at least $n+1-p$ distinct zeros in the open interval $(-1,1)$. But $T_{n}{ }^{(p)}(x)$ and $S_{n}(p)(x)$ are linearly independent solutions of the differential equation (3), so by Sturm's well known theorem the zeros of $T_{n}^{(p)}(x)$ and $S_{n}^{(p)}(x)$ separate one another. It follows that $S_{n}(p)(x)$ has exactly $n+1-p$ zeros in the interval $(-1,1)$, all of which are simple, $(p=1,2,3, \cdots, n)$. For the case $p=n+1$ we see from (3) that $S_{n}^{(n+1)}(x)$ is a solution of the differential equation

$$
\left(1-x^{2}\right) \frac{d}{d x} S_{n}^{(n+1)}(x)-(2 n+1) x S_{n}^{(n+1)}(x)=0 .
$$

Suppose that $S_{n}^{(n+1)}(x)$ had at an interior point $x_{0}$ a zero of order $k$, $(k \geqq 1)$. Then, from the differential equation, $d S_{n}{ }^{(n+1)}(x) / d x$ would have a zero of order $k$ (or of order $k+1$ if $x_{0}=0$ ). This would demand that $S_{n}^{(n+1)}(x)$ have a zero of order $k+1$ (or of order $k+2$ if $x_{0}=0$ ), in contradiction to the assumption. This proves that $S_{n}^{(n+1)}(x)$ cannot have a zero.

LEMma 2. In the expansion

$$
M_{p}(x)=\sum_{k=0}^{\infty} a_{2 k} x^{2 k},
$$

valid for $|x|<1,(1 \leqq p \leqq n)$, all the coefficients are greater than zero.

Proof. The statement is evidently true for $p=1$, since, by definition,

$$
M_{1}(x)=\left(T_{n}^{\prime}(x)\right)^{2}+\left(S_{n}^{\prime}(x)\right)^{2}=n^{2} /\left(1-x^{2}\right) .
$$

We now proceed by induction. Let

$$
\begin{aligned}
M_{p}(x) & =\sum_{k=0}^{\infty} a_{2 k} x^{2 k}, \\
M_{p+1}(x) & =\sum_{k=0}^{\infty} b_{2 k} x^{2 k} .
\end{aligned}
$$


On substituting these power series in the differential equation (6) we obtain between the coefficients the relation

$$
k b_{2 k}+k\left(n^{2}-p^{2}\right) a_{2 k}=(2 p+k) b_{2 k-2} .
$$

Suppose that every coefficient $a_{2 k}$ is greater than zero. From the relation (7) we see that if, for a particular $k, b_{2 k}$ is positive, then $b_{2 k-2}$ is also positive; and by repeated use of this relation each of the coefficients $b_{2 k-4}, b_{2 k-6}, \cdots, b_{2}, b_{0}$ is positive. Thus if $b_{2 k}$ is greater than zero, then the same is true of each of the preceding coefficients. The functions $S_{n}^{(p+1)}(x)$ and $M_{p+1}(x)$ are unbounded in the interval $(-1,1)$ since $S_{n}^{\prime}(x)$ becomes infinite as $x \rightarrow \pm 1$. This shows, since $M_{p+1}(x)$ is non-negative, that there are arbitrarily large values of $k$ for which $b_{2 k}$ is greater than zero. But if one coefficient is positive, we have shown that all the preceding ones are also positive, and it follows that all $b_{2 k}$ are positive. This completes the induction.

By definition (1), $T_{n}(1)=1$, and by (3)

$$
(2 p+1) T_{n}^{(p+1)}(1)=\left(n^{2}-p^{2}\right) T_{n}^{(p)}(1) .
$$

From this we find by induction that

$$
T_{n}^{(p)}(1)=\frac{n^{2}\left(n^{2}-1^{2}\right)\left(n^{2}-2^{2}\right) \cdots\left(n^{2}-(p-1)^{2}\right)}{1 \cdot 3 \cdot 5 \cdot \cdots \cdot(2 p-1)} .
$$

Theorem 1. Let $f(x)$ be a polynomial of degree $n$ or less with real coefficients such that

$$
|f(x)| \leqq 1, \quad-1 \leqq x \leqq 1,
$$

and suppose that $f(x) \not \equiv \gamma T_{n}(x)$. Then for every real $\alpha$ the first $n$ derivatives of the function

$$
\cos \alpha T_{n}(x)+\sin \alpha S_{n}(x)-f(x)
$$

can have only simple zeros in the interval $(-1,1)$.

Proof. There is no loss of generality in supposing that $0 \leqq \alpha<\pi$. Let

(9) $\left\{\begin{array}{l}R(x)=\cos \alpha T_{n}(x)+\sin \alpha S_{n}(x)=\cos (n \theta-\alpha), \quad x=\cos \theta \\ R^{\prime}(x)=n \sin (n \theta-\alpha) / \sin \theta .\end{array}\right.$

Then at the points where $R(x)$ vanishes we have

$$
\left(R^{\prime}(x)\right)^{2}=\frac{n^{2}}{1-x^{2}}
$$


hence, by Bernstein's theorem,

$$
\left|f^{\prime}(x)\right|<\left|R^{\prime}(x)\right|
$$

at these points. If $\alpha \neq 0$, this inequality is also true in a neighborhood of the points -1 and +1 , for $S_{n}^{\prime}(x)$ becomes infinite here while $T_{n}^{\prime}(x)$ and $f^{\prime}(x)$ are bounded in the whole interval. We now distinguish two cases, $\alpha=0$ and $\alpha>0$.

CASE I. $\alpha=0$.

In this case $R(x) \equiv T_{n}(x)$, and the function considered reduces to

$$
T_{n}(x)-f(x)
$$

which is a polynomial of degree $n$ or less, not identically zero. Then by (10)

$$
T_{n}^{\prime}(x)-f^{\prime}(x)
$$

is alternately plus and minus at the $n$ points where $R(x)$ vanishes; so it has at least $n-1$ distinct zeros. Using Rolle's theorem we see that $T_{n}{ }^{(p)}(x)-f^{(p)}(x)$ has at least $n-p$ distinct zeros, $(1 \leqq p \leqq n)$, and it can have no others, as it is a polynomial of degree $n-p$. Thus all its zeros are simple and Theorem 1 is true for the case $\alpha=0$.

CASE II. $0<\alpha<\pi$.

We are going to show first that in this case the function

$$
R^{\prime}(x)-f^{\prime}(x)
$$

has at least $n$ distinct zeros.

If $\alpha=\pi / 2$, then $R(x) \equiv S_{n}(x)$, and we see that at the $n-1$ zeros of $S_{n}(x)$ in the interior of $(-1,1)$ and in a neighborhood of the two end points the inequality (10) is satisfied. Thus, $S_{n}^{\prime}(x)$ is alternately plus and minus at $n+1$ successive points where (10) is true; so the function

$$
R^{\prime}(x)-f^{\prime}(x) \equiv S_{n}^{\prime}(x)-f^{\prime}(x)
$$

has at least $n$ distinct zeros in $(-1,1)$.

If $\alpha \neq \pi / 2$, then either $0<\alpha<\pi / 2$ or $\pi / 2<\alpha<\pi$, and as the two cases are essentially the same we shall consider only the case $0<\alpha<\pi / 2$. From relation (9) we see that $R(x)$ vanishes at the $n$ points $\theta=\left[\left(k+\frac{1}{2}\right) \pi+\alpha\right] / n,(k=0,1, \cdots, n-1)$, in $(-1,1)$. Selecting these $n$ points and one point from a small neighborhood of $x=1$, we have $n+1$ points in $(-1,1)$ at which the inequality $(10)$ is satisfied. From the relation (9) it is seen that $R^{\prime}(x)$ is alternately plus and minus at these $n+1$ successive points. Then the function 


$$
R^{\prime}(x)-f^{\prime}(x)
$$

is alternately plus and minus at $n+1$ points and so has at least $n$ distinct zeros.

Thus if $0<\alpha<\pi$, the function

$$
R^{\prime}(x)-f^{\prime}(x)
$$

has at least $n$ distinct zeros in $(-1,1)$. Using Rolle's theorem one shows that

$$
R^{(p)}(x)-f^{(p)}(x)
$$

has at least $n+1-p$ distinct zeros. If it had one multiple zero, then $R^{(p+1)}(x)-f^{(p+1)}(x)$ would have at least $n+1-p$ distinct zeros, and finally

$$
R^{(n+1)}-f^{(n+1)}
$$

would have at least one zero. Since $f(x)$ and $T_{n}(x)$ are polynomials of degree $n$ at most this means that $S_{n}^{(n+1)}(x)$ has at least one zero at some interior point of $(-1,1)$; but by Lemma 1 this is impossible. This completes the proof of Theorem 1.

Theorem 2. For any polynomial $f(x)$ of degree $n$ or less, the inequality $|f(x)| \leqq 1$ in $(-1,1)$ implies

$$
\left|f^{(p)}(x)\right|^{2} \leqq M_{p}(x), \quad-1 \leqq x \leqq 1 ; p=1,2,3, \cdots, n,
$$

and the equality can occur only if $f(x) \equiv \gamma T_{n}(x)$.

Proof. For simplicity we shall suppose that $f(x) \not \equiv \gamma T_{n}(x)$. We shall consider first the case in which all the coefficients of $f(x)$ are real. Suppose that at some point $x_{0},\left(-1<x_{0}<1\right)$, we have

$$
\left|f^{(p)}\left(x_{0}\right)\right|^{2} \geqq M_{p}\left(x_{0}\right)
$$

for some $p,(1 \leqq p \leqq n)$. Consider the function

where $R(x)$ is of the form

$$
R(x)+\lambda f(x),
$$

$$
R(x)=\cos \alpha T_{n}(x)+\sin \alpha S_{n}(x),
$$

and $\alpha$ and $\lambda$ are real constants to be determined. We shall show that if (11) is true, then real $\alpha$ and $\lambda$ exist, $(-1 \leqq \lambda \leqq 1)$, so that at the point $x_{0}$ the $p$ th derivative of the function $R(x)+\lambda f(x)$ has a double zero.

Let $\alpha$ be chosen so that the relation

$$
R^{(p+1)}\left(x_{0}\right) f^{(p)}\left(x_{0}\right)-R^{(p)}\left(x_{0}\right) f^{(p+1)}\left(x_{0}\right)=0
$$


is satisfied. This is always possible for, on expressing $R^{(p)}(x)$ and $R^{(p+1)}(x)$ by use of equation (12), the relation (13) is equivalent to an equation of the form

$$
a \cos \alpha+b \sin \alpha=0,
$$

where $a$ and $b$ are real, and this has a solution.

Having chosen $\alpha$ we see from Cauchy's inequality that

$$
\left|R^{(p)}(x)\right|^{2} \leqq\left\{\cos ^{2} \alpha+\sin ^{2} \alpha\right\}\left\{\left(T_{n}^{(p)}(x)\right)^{2}+\left(S_{n}^{(p)}(x)\right)^{2}\right\} \equiv M_{p}(x) .
$$

Hence (supposing that inequality (11) is true) we choose $\lambda$, $(-1 \leqq \lambda \leqq 1)$, so that at the point $x_{0}$

$$
R^{(p)}\left(x_{0}\right)+\lambda f^{(p)}\left(x_{0}\right)=0 .
$$

Substituting this in equation (13), we have

$$
f^{(p)}\left(x_{0}\right)\left\{R^{(p+1)}\left(x_{0}\right)+\lambda f^{(p+1)}\left(x_{0}\right)\right\}=0
$$

and this means that the second factor is zero. Thus the pth derivative of the function

$$
R(x)+\lambda f(x)
$$

has a double zero at the point $x_{0}$, but by Theorem 1 this is impossible. The contradiction proves Theorem 2 in the case in which all the coefficients of $f(x)$ are real.

Now allow $f(x)$ to have complex coefficients, ${ }^{*}$ and choose a real con$\operatorname{stant} \beta$ so that, at a point $x_{0}$ arbitrarily chosen in $(-1,1)$,

is real. Writing

$$
e^{i \beta} f^{(p)}\left(x_{0}\right)
$$

$$
e^{i \beta} f(x)=f_{1}(x)+i f_{2}(x),
$$

where $f_{1}(x)$ and $f_{2}(x)$ have real coefficients, we see that if $f_{1}(x)$ were of the form $f_{1}(x) \equiv \gamma T_{n}(x)$, then $f_{2}(x)$ would vanish at the $n+1$ points where $T_{n}(x)= \pm 1$ and so would vanish identically. Then $f(x)$ itself would be of the form $f(x) \equiv \gamma T_{n}(x)$; but we have supposed that this is not the case, so $f_{1}(x) \not \equiv \gamma T_{n}(x)$. We have already proved that Theorem 1 applies to the polynomial $f_{1}(x)$, and since $e^{i \beta} f^{(p)}\left(x_{0}\right)=f_{1}^{(p)}\left(x_{0}\right)$, it fol= lows that

$$
\left|f^{(p)}\left(x_{0}\right)\right|^{2}=\left|f_{1}^{(p)}\left(x_{0}\right)\right|^{2}<M_{p}\left(x_{0}\right) .
$$

* This method of extending inequalities to polynomials with complex coefficients has been used by S. Bernstein, Leçons sur les propriétés extrémals et meilleure approximation des fonctions analytiques d'une variable réelle, Paris, 1926, p. 45. 
This completes the proof of Theorem 2 .

Lemma 3. Let $f(x)$ be a polynomial of degree $n$ or less, such that

$$
|f(x)| \leqq 1
$$

in $(-1,1)$. If $x_{0}$ is a point of $(-1,1)$ which lies either to the left or to the right of all zeros of $S_{n}^{(p)}(x)$, then

$$
\left|f^{(p)}\left(x_{0}\right)\right| \leqq\left|T_{n}^{(p)}\left(x_{0}\right)\right|,
$$

and the equality can occur only if $f(x) \equiv \gamma T_{n}(x)$.

Proof. Let $a_{k},(k=1,2,3, \cdots, n+1-p)$, be the zeros of $S_{n}{ }^{(p)}(x)$ in the interval $(-1,1)$. Let $\phi(x)$ be a polynomial of degree $n+1-p$ which vanishes at these $n+1-p$ points,

$$
\phi(x)=\left(x-a_{1}\right)\left(x-a_{2}\right) \cdots\left(x-a_{n+1-p}\right) .
$$

Then using the Lagrange interpolation formula we have, since $f^{(p)}(x)$ is a polynomial of degree $n-p$ or less,

$$
f^{(p)}(x)=\phi(x) \sum_{k=1}^{n+1-p} \frac{f^{(p)}\left(a_{k}\right)}{\phi^{\prime}\left(a_{k}\right)\left(x-a_{k}\right)},
$$

and there is a similar expression for $T_{n}{ }^{(p)}(x)$. The zeros of $S_{n}^{(p)}(x)$ and $T_{n}^{(p)}(x)$ interspace one another, so $T_{n}^{(p)}(x)$ is alternately plus and minus at the successive zeros of $S_{n}{ }^{(p)}(x)$, and it is easily seen that $\phi^{\prime}(x)$ alternates in sign at successive zeros of $\phi(x)$. Thus all the numbers

$$
\frac{T_{n}^{(p)}\left(a_{k}\right)}{\phi^{\prime}\left(a_{k}\right)}
$$

are of the same sign, $(k=1,2,3, \cdots, n+1-p)$. Moreover, by Theorem 2, we have at the zeros of $S_{n}^{(p)}(x)$

$$
\left|f^{(p)}\left(a_{k}\right)\right|^{2} \leqq M_{p}\left(a_{k}\right)=\left(T_{n}^{(p)}\left(a_{k}\right)\right)^{2} .
$$

Now let $x_{0}$ be a point which lies to the right of all zeros of $S_{n}^{(p)}(x)$, so that $x_{0}-a_{k}>0,(k=1,2, \cdots, n+1-p)$. Then it follows immediately that

$$
\begin{aligned}
\left|f^{(p)}\left(x_{0}\right)\right| & \leqq\left|\phi\left(x_{0}\right)\right| \sum\left|\frac{f^{(p)}\left(a_{k}\right)}{\phi^{\prime}\left(a_{k}\right)\left(x_{0}-a_{k}\right)}\right| \\
& \leqq\left|\phi\left(x_{0}\right)\right| \sum\left|\frac{T_{n}^{(p)}\left(a_{k}\right)}{\phi^{\prime}\left(a_{k}\right)\left(x_{0}-a_{k}\right)}\right|=\left|T_{n}^{(p)}\left(x_{0}\right)\right| .
\end{aligned}
$$

The equality can occur throughout (15) only if the equality is true 
in (14), and by Theorem 2 this is true only if $f(x) \equiv \gamma T_{n}(x)$. This proves Lemma 3 in the case where $x_{0}$ lies to the right of all zeros of $S_{n}{ }^{(p)}(x)$, and the same method is available if $x_{0}$ lies to the left of all zeros of $S_{n}{ }^{(p)}(x)$.

We can now prove the theorem of W. Markoff.

MARKOFF's THEOREM. If $f(x)$ is a polynomial of degree $n$ or less, the inequality $|f(x)| \leqq 1,(-1 \leqq x \leqq 1)$, will imply

$$
\left|f^{(p)}(x)\right| \leqq \frac{n^{2}\left(n^{2}-1^{2}\right)\left(n^{2}-2^{2}\right) \cdots\left(n^{2}-(p-1)^{2}\right)}{1 \cdot 3 \cdot 5 \cdots \cdot(2 p-1)},-1 \leqq x \leqq 1 \text {, }
$$

for $p=1,2,3, \cdots, n$. The equality can occur only at $x= \pm 1$ and here only if $f(x) \equiv \gamma T_{n}(x)$.

Proof. By Theorem 2 we see that

$$
\left|f^{(p)}(x)\right|^{2} \leqq\left(T_{n}^{(p)}(x)\right)^{2}+\left(S_{n}^{(p)}(x)\right)^{2} \equiv M_{p}(x),
$$

and in Lemma 3 we have shown that $M_{p}(x)$ increases monotonically in $(0,1)$. Let $a,(|a|<1)$, be the zero farthest to the right of $S_{n}{ }^{(p)}(x)$ in the interval $(-1,1)$; then in the interval $(-a, a)$ we have

$$
\left|f^{(p)}(x)\right|^{2} \leqq M_{p}(x) \leqq M_{p}(a)=\left(T_{n}^{p)}(a)\right)^{2} .
$$

We know that $T_{n}{ }^{(p)}(x)$ increases monotonically in the interval $(a, 1)$, since, by Lemma 1 , its zeros lie in the interval $(-a, a)$. Moreover $T_{n}^{(p)}(x)$ is either an odd or an even function of $x$, hence the maximum of its absolute magnitude in the intervals $(-1,-a)$ and $(a, 1)$ occurs at $x= \pm 1$. Then if $x_{1}$ lies in $(-a, a)$,

$$
\left|f^{(p)}\left(x_{1}\right)\right| \leqq\left|T_{n}^{(p)}(a)\right|<\left|T_{n}^{(p)}(1)\right| .
$$

If $x_{2}$ lies in either of the intervals $(-1,-a),(a, 1)$, we have from Lemma 3

$$
\left|f^{(p)}\left(x_{2}\right)\right| \leqq\left|T_{n}^{(p)}\left(x_{2}\right)\right| \leqq\left|T_{n}^{(p)}(1)\right|,
$$

and the equality can occur only at $x_{2}= \pm 1$. Thus, if $x$ lies in the interval $(-1,1)$, we have the inequality

$$
\left|f^{(p)}(x)\right| \leqq\left|T_{n}^{(p)}(1)\right|,
$$

and the equality can occur only at $x= \pm 1$, and here only if $f(x) \equiv \gamma T_{n}(x)$. The explicit expression for $T_{n}^{(p)}(1)$ obtained in (8) furnishes the final step in the proof of Markoff's theorem.

Purdue University 